\title{
Correspondence
}

Editor: Ian Pullen

Contents: Molecular genetics and human disease/ Non-psychotic psychiatric disorder after childbirth/ 'Biological' treatment/Journal supplements/Prevalence of dementia/History of depressive disorder/ Supportive analytical psychotherapy/Ethnic density and schizophrenia/Treatment of suicidal manic depression/Chronic depression/Failure to convulse with ECT/'Good old days' of psychiatry

\section{Molecular Genetics and Human Disease}

SIR: I would like to follow Dr Pelosi's lead (Journal, October 1988, 153, 570) in breaking the deafening silence surrounding the ethical implications of the new genetics. Psychiatry stands on the threshold of its most exciting era. The chance to increase understanding of our most devastating mental disorders and perhaps to develop new treatments is open to us. This exists alongside possibilities for serious misuses by clinicians with the most laudable of motives, who may be tempted to step beyond their duty to relieve the suffering of the individual into the realm of attempting to alter the composition of society by applying eugenic principles. When scientists in the first half of the century discovered atomic energy many were immediately aware of its potential for both good and ill. Molecular genetic technology is no less awesome, and it behoves our profession to use some foresight in considering its potential.

Dr Pelosi raises Huntington's disease as a case in point: a condition whose gene acts with ruthless inevitability quite unlike the gene or genes underlying commoner psychiatric disorders. Those ethical dilemmas which may arise in relation to the latter will be magnified and even more complicated. Open debate on this topic is therefore mandatory. Furthermore, we should not make the mistake of equating the diagnostic clarity promised by genetic research with moral certainty. Aubrey Lewis, the most influential British psychiatrist of recent times, was himself rather sympathetic to the eugenic movement. $\mathrm{He}$ argued: "Not only eugenists but all compassionate and reflective people must surely be concerned at an increase in the number of children born to parents who are themselves affected by a prolonged mental illness or who are destined to become mentally ill." (Lewis, 1958). In summary, he warned: "We do not know enough to warrant our making firm predictions or advocating celibacy and childlessness to outwardly healthy people who seem predisposed to mental illness."

We may soon know enough to make much firmer predictions than was hitherto imaginable. History will judge if we use this knowledge wisely.

ANTHONY S. DAVID

Institute of Psychiatry

De Crespigny Park

London SE5 8 AF

\section{Reference}

LEWIs, A. (1958) Fertility and mental illness. Eugenics Review, 50, 91-106.

\section{Non-Psychotic Psychiatric Disorder After Childbirth}

SiR: Cooper et al (Journal, June 1988, 152, 799-806) are to be congratulated on their careful controlled study of the prevalence of non-psychotic psychiatric disorder during the first year after childbirth. However, the finding that the prevalence in the Oxford mothers was not greater than in the Edinburgh controls is not in conflict with my own study (Pitt, 1968) to which they refer; I was not looking at prevalence, but incidence.

I had assumed that many women's mental health might actually benefit from the acquisition of a wanted or not unwelcome baby, and that those who responded adversely would be a minority. Indeed, there was a small but significant drop in scores on my questionnaire for symptoms of anxiety and depression at the time of childbearing between the third/fourth months of pregnancy and the puerperium. However, $10.8 \%$ at least (Neugebauer (1983) calculated that this was an almost $50 \%$ underestimate!) developed within two months of giving birth a state of depression (most often, I found, conforming to the 'neurotic' pattern - I rather regret having used the alternative term 'atypical') unusual for them, disabling, and thoroughly unwelcome. I thought 
that this incidence was unexpectedly high, and the findings of Dr Cooper et al confirm this.

The overall incidence of psychiatric disorder in their study was $151 / 1000$ in the post-natal year, which is only $14 \%$ higher than in a rather unsatisfactory Edinburgh control group aged 18-65 (not many women give birth after 50). However, as the authors concede, "there was a tendency for the onset of psychiatric disorder to arise in the first 3 months after delivery rather than evenly throughout the postpartum year". Indeed there was: $24 \%$ of the incidence was within a month of childbirth, $40 \%$ within 3 months and only $27 \%$ in the last 6 months of the post-natal year.

On the evidence presented it would be premature to write the obituary of post-natal depression.

St Mary's Hospital Medical School BRICE PITT

Paddington

London W2

\section{References}

Neugebauer, R. (1983) Rate of depression in the puerperium. British Journal of Psychiatry, 143, 421-422.

PITT, B. (1968) "Atypical" depression following childbirth. British Journal of Psychiatry, 114, 1325-1335.

SIR: Without doubt the study by Cooper et al (Journal, June 1988, 152, 799-806) is one of the most thorough into the vexed question as to whether childbirth contributes to a genuine increase in nonpsychotic morbidity. The results in fact showed no significant difference between the level of such morbidity in a group of puerperal women and that in a non-puerperal control group. The controls were not studied directly by the authors but were a subset of a general population sample of women studied by Surtees et al (1983) in Edinburgh. They were non-puerperal in that they had had no pregnancy or delivery during the previous year. The problem with this control group is that it may have contained women in their second or even third postnatal year still suffering from disorders which had had a postnatal onset.

The authors themselves acknowledge that such disorders may pursue a chronic course, and they refer to Pitt (1968), who found that $3.9 \%$ of his total sample of 305 women had depressive disorders which showed little or no improvement a year after initial assessment. Of particular relevance for an Edinburgh population is the finding reported by Wrate et al (1985) of a 3-year follow-up study of 103 mothers. Of 11 with postnatal depression, $7(6.8 \%$ of the total sample) had disorders which lasted at least until the end of the first postnatal year and 2 mothers had disorders lasting for more than two years. Furthermore, Dr Cooper et al show that about one-third of their own puerperal cases are detectable at twelve months postpartum, and they presumably remained cases for at least part of the second postpartum year.

In clinical practice one certainly does see women in the second or, to a lesser extent, third year postpartum with non-psychotic disorders which have pursued a chronic persistent or relapsing course since delivery. Such women should be excluded from the control group in order to derive a better estimate of the psychiatric morbidity among the general female population of childbearing age; one which is independent of the effect of childbirth, although not independent of the effect of the stresses of childcare, and hence suitable for use as a control value.

Paul-Allan Dewsnap

Grorge STEIN

Farnborough Hospital

Farnborough Common

Orpington

Kent BR6 8ND

\section{References}

PITT, B. (1968) "Atypical" depression following childbirth. British Journal of Psychiatry, 114, 1325-1335.

Surtees, P. G., Dean, C., Ingham, J. G., el al (1983) Psychiatric disorder in women from an Edinburgh community: associations with demographic factors. British Journal of Psychiatry, 142, 238-246.

Wrate, R. M., Rooney, A. C., Thomas, P. F., el al (1985) Postnatal depression and child development: a three year follow-up study. British Journal of Psychiatry, 146, 622-627.

\section{'Biological' Treatment}

SIR: In his letter (Journal, September 1988, 153, 405) commenting on my use of the word 'biological' in my article (Journal, May 1988, 152, 657-659) on the prediction of response of depressed patients to treatment, Dr Van Kempen asks the question, "why not 'drug' or 'pharmacological' treatment?" The answer is to be found in the first paragraph on page 659, where it is indicated that 23 patients received ECT either alone or in combination with drugs. Perhaps I could have used the word 'physical' to cover this combination of treatments, but in considering the aetiology and treatment of psychiatric disorders it is accepted practice to categorise the factors as psychological, social, and biological.

On the broader issue of the meaning of the word 'biological', I take the point that it is generally held to imply a relation to the science of life in general, but the Shorter Oxford English Dictionary (3rd edition, 1973) gives two meanings: (a) "the study of human life and character", which is indicated as obsolete, 